\title{
Face Age Estimation and the Other-race Effect
}

\author{
Oluwasegun Oladipo ${ }^{1}$ \\ Department of Computer and \\ Information Science, Covenant \\ University, Ota, Ogun State; CITM \\ Yaba College of Technology \\ Yaba, Lagos. Nigeria
}

\author{
Elijah Olusayo Omidiora ${ }^{2}$ \\ Department of Computer Science \\ and Engineering, LAUTECH \\ Ogbomoso, Oyo State \\ Nigeria
}

\author{
Victor Chukwudi Osamor ${ }^{3}$ \\ Department of Computer and \\ Information Sciences \\ Covenant University, Ota \\ Ogun State, Nigeria
}

\begin{abstract}
Age estimation is an automated method of predicting human age from 2-D facial feature representations. The majority of studies carried out in this research area use the FG-NET and MORPH 2 databases to train and test developed systems, which are lacking in black-face content. Most age frauds are perpetuated in the sub-Saharan African region due to the unavailability of an official database and unregistered births in the rural areas. The issues of unverified age in the region made it possible for under-age voters, under-age drivers, and the engagement of over-aged sportsmen. The other-race effect could reduce the performance of face recognition techniques, which could make techniques that work for white faces underperform when deployed for use in the predominately black face region. This study examines the other-race effect on face-based age estimation by analyzing the accuracy of an age estimation system trained with predominantly black faces against the same age estimation system trained with predominately white faces. The developed age estimation system uses a genetic algorithmartificial neural network classifier and local binary pattern for texture and shape feature extraction. A total of 170 black faces were used for system testing. The result showed that the age estimation system trained with the predominantly black face database (GA-ANN-AES-855) outperformed the system trained with predominantly white faces (GA-ANN-AES-255) on testing with the aforementioned black face samples. The results obtained from the simulation were further subjected to inferential statistics, which established that the improvement in the correct classification rate was statistically significant. Hence, the otherrace effect affects face-based age estimation systems.
\end{abstract}

Keywords-Component; face recognition; age estimation; other-race effect

\section{INTRODUCTION}

Computer-based age estimation is an automated way of predicting human age from 2-D features extracted from the face image. Most of the age fraud in recent days has been committed in Africa. The fraud goes unnoticed due to factors such as the unavailability of the national database of birth registration; some people give birth in unaccredited maternity centers and do not have the border to obtain birth certificates; and the acceptance of affidavit without questioning the authenticity [1].

Age validation and verification is one of the areas with least technological penetration in Africa. There is practically no popular IT-based system or tool to automatically verify an age claim. This made it possible for underage wards to beat systems and have access to documents like driver's license and voter cards. Over-aged sportsmen still go unnoticed and people apply for jobs that are meant for people of lower ages. These are possible because of prevalent situation in Africa such as: Unclarified sworn to age affidavit that is not most-of -the-time confirmed, Birth certificate that was never collected at the point of giving birth to a child in the hospitals. Also, in subSaharan region of Africa, most people were not born in hospitals; as a result, most births were not documented by the National population commission [2].

In view of the above, most of the age claims made by the people in this region may not be true and are quite unreliable. Hence, there is a great need for an efficient and robust system for black-face age estimation and validation [2].

According to [3], most age estimation systems developed use MORPH II and FG-NET face databases, and these databases are scarcely populated with black face images, not considering the other-race effect. The other-race effect could make a face recognition technique that performs well with a specific race underperform when deployed for testing with faces of other races [4]. Hence, this study is aimed at examining the other-race effect on face-based age estimation. Memory depiction in humans and allied researches buttress the significance of other race in face recognition. The way human memory is depicted from literatures supports the consequence of the other race effect. This is evident in the phrasal saying; "they all look alike to me". Expanding this shows that some discriminating features makes identifying humans from the same race easily encoded and retrievable rather than the other race. The complications in the representation of human memory are what makes it seem like they all look alike despite being different individuals [5].

This property exhibited in humans can also be as a result of social prejudice, less frequent communication and little familiarity shared with people from different origin. During growth, a child's memory representation develops its ability to discriminate faces of his own race. In retrospect, this however mitigates the discernment capability of people within other race [6].

Theoretically, this can be explained from the principle of feature selection and associated processes. This selection begins during the child's development phase and procedure for facial identity discernment becomes memorably registered on a daily contact basis in a child. The aftermath is a deduction of excellent facial representation and encoding of faces which are seldom contacted and seen [7]. 
The other race effect could influence a computer-based recognition task such as age estimation, as it involves rigorous training procedure to aptly portray human faces. Also, there is a limited database on demographic category coverage for training different algorithms to identify the faces of individuals based on their respective racial backgrounds. Hence, an age estimation system that works well with white faces or other race might underperform when deployed for use with black faces [8][5].

The following are the objectives of the study:

- To develop a database of predominantly black faces.

- Training and testing of genetic algorithm-artificial neural network-based age estimation using the developed database.

- Performance evaluation of the age estimation system side by side with one trained with fewer black faces using correct classification.

The study is organized into five sections. Section I introduces the study with a brief discussion of the key words and also outlines the study objectives. Section II describes various related works in the area of face-based age estimation. Section III shows the methodology used in the study with their algorithms, while Section IV discusses the simulation result. The study is concluded in section $\mathrm{V}$ with recommendations for further studies.

\section{RELATED WORK}

Age prediction is often articulated in two categories. It's either categorized as a classification problem when a face is computed to be associated with one of $\{\mathrm{xa} 1, \mathrm{xa} 2, \mathrm{xa} 3 \ldots \mathrm{xaN}\}$ specified age group or as a regression problem which aims at evaluating age as a scalar xa $\in$ R. [9][10], proffer a classification centered model which proceeds in two steps. The first step involved classifying faces to genders and race, while age is in turn estimated for the different and races. An ordinal classification model was deployed for age prediction in [11] [12]. Positioned decision boundaries are used in splitting the facial features into groups depending on their relational order. The ages are deduced by combining organized references from the positioned decision boundaries.

In the same vein, Han and Otto [13] [14] employed a hierarchical model for age prediction and also examined the effect of aging on features extracted from a 3-D face image. [15] Formulated a system for crowd density and age prediction despite spare and unbalanced datasets, which are a common limitation to age estimation research. The developed system could harness the cumulative attribute approach to learning a regression model in the presence of face image dataset imbalances.

In [16], the author study offered a sparse regression approach, trained with the Face Recognition Grand Challenge database. The resulting model was tested using images in the FG- NET database. In [17], their study employed the use of deep learning architecture together with manifold learning for age estimation. The convolutional network was deployed for feature extraction and face aging features were obtained at various layers of the system developed rather than just the top layer. The authors in [18] and [19] also pursue the use of a convolutional neural network (CNN) to increase the accuracy of the age estimation system.

The author in [20], developed an age estimation system using a back propagation artificial neural network trained with the FG-NET database. In this work, face images were classified into eight (8) age groups and principal component analysis was used for appearance and texture information representation.

The author in [21], examined the performance of age estimation systems developed using back propagation artificial neural network and self-organizing feature maps, which is an unsupervised learning paradigm neural network. The study employed principal component analysis for feature extraction and statistically examined which of the two aforementioned system performed better. The study deduction showed that the self-organizing feature map performed better than the back propagation trained artificial neural network.

The author in [22] showed that the performance of age estimation system can be improved by using large scale databases with deep learning algorithms. The study proposed a ranking $\mathrm{CNN}$ model that consists of basic $\mathrm{CNN}$ combined in series. The CNN content is trained with ordered age labels, and the final output is an aggregation formed from the binary output of constituent CNNs. It was concluded that ranking$\mathrm{CNN}$ has the ability to outperform conventional CNN models.

The author in [23], deployed cumulative hidden layer approach to combat the issue of image dataset imbalances which are inherent in large databases. In the model learned age features using faces from neighboring ages uses a pair-wise relative signal in the supervision of the comparative ranking layer. The aging feature learning is fostered by the implemented ranking layer and also improves the age estimation of the overall model.

The author in [24] proposed a multi modal approach to age and gender estimation by using face images and speech recognition for age estimation. Two joint deep neural networks were trained with both appearance and depth information extracted from the face image. This is done alongside with the mel frequency cepstral coefficient extracted from speech samples. A novel cost function was developed to fine tune the joint deep neural network to ensure better accuracy and reduce the overhead of over fitting.

The author in [25], noted that fluctuation might result from deploying conventional convolutional neural network to face images in a video frame for age estimation. Furthermore, an attention mechanism was employed in addition to the convolutional network. This models an attention chunk which contains an aggregated feature space which is in turn presented as an encoded feature for age estimation. The stabilization of the frames is achieved using a novel loss function in order to achieve better age estimation of images in the frame.

The author in [26] used a variant of auto encoder for age, gender and race classification. In [27] their study, developed a hybrid system from the combination of CNN and Extreme Learning Machine (ELM). The CNN was adopted for age- 
related feature extraction from the face images used while the ELM was deployed to classify into various age brackets. The study used the popular database MORPH II and the audience database for the training and testing of the developed system. The author in [28] leveraged the interrelationship of secondary demographic evidence such as race and gender in training a cascaded structured model. The secondary information assists in learning all the frameworks which are embedded in the parent network and sub-networks. This improved the accuracy of age prediction when compared to the previous age estimation system.

The author in [29] adopted the fusion of Local Discriminant Analysis (LDA), LBP, and Gabor filters for appearance, shape, and texture representation of the face image for age estimation. The study also used a combination of Support Vector Machine (SVM) and ANN to classify the age of the face into various age brackets. The ensemble was guided by the majority voting scheme and a computed age label was assigned from the combination of global and section-built matchers.

The author in [30] took cognizance of images taken in an uncontrolled environment and real-life situations using mobile phones. Such images have a degraded quality due to the phone camera's resolution. The images are fed into a conditional generative adversarial network (GAN) model to generate a reconstructed high-resolution version. The study used CNN for age estimation with PAL and MORPH face image databases for training and testing. Hasan \& Mahdi [31] in their study used SVM as a classifier with LBP and FSM feature extraction techniques to represent face images. The two-way feature selection helped improve the performance of the age estimation system.

It is essential to keep track of the trends in research on automatic age prediction. Deep learning approaches seem promising but are known not to perform well with insufficient data [22]. Face image data collection is tedious in African contests. People over the age of 40 (fourth) find it difficult to enroll their faces. They are concerned that their true age will be revealed and will conflict with their official record at work, as some claim an age that is lower than their true age in order to secure available jobs for lower ages.

In order to ensure the limited black face dataset does not impair the performance of the developed system, the study uses a genetic algorithm modified back propagation trained artificial neural network-based classifier for age group estimation task. It also used selected data from the FG-NET database to complement the locally sourced data.

\section{Methodology}

Human faces are undoubtedly affected by physical development and the aging process. This facial alteration varies from person to person and is a consequence of a variety of factors like ancestry, health, lifestyle, gender, and race [28]. This study developed an age estimation system using a Genetic Algorithm (GA)-Artificial Neural Network (ANN). Local binary patterns were used for feature extraction in order to encode face images, which were trained using the GA-ANN module. The architecture of the developed system is shown in Fig. 1.

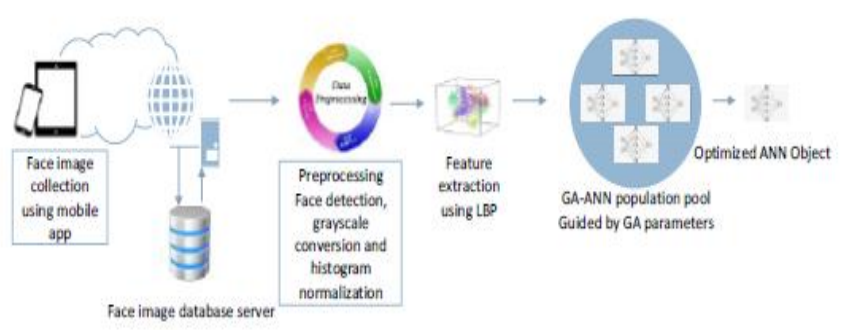

Fig. 1. The Developed Architecture.

It should be noted that the developed system used a shallow learning approach as opposed to the deep learning paradigm due to the limited number of face images in the database. The deep learning approach is known to have performance degradation issues with small datasets.

In order to examine the other-race effect in age estimation, the developed GA-ANN-based age estimation system was trained with the predominantly black face database developed for this study and also tested with 170 black face images. The result of this simulation was compared side by side with the result of a simulation done with the age estimation system trained with fewer black faces. The performance matrix used in this study is the Correct Classification Rate (CCR) of the age estimation system. The philosophy of the study is to examine if there is a significant difference in the performance of an age estimation system trained with more black faces when it is deploy for use with black faces. A One way ANOVA statistical tool was used for this purpose.

\section{A. Face Image Collection}

The face image collection was done using a custom built mobile application. The sequence diagram for the mobile app developed is shown in Fig. 2. The mobile application was developed to ease data collection from remote and distant locations. Fig. 3 displays the data collection module of the app. The mobile app is made available to voluntary candidates on Google Drive. The application was developed using HTML5, JavaScript, and CSS to ensure that it could be deployed for use across various mobile phone operating systems after compiling using CORDOVA. As a result, data sourcing was not restricted to specific locations and distances. The FG-NET facial database was used to complement the locally sourced data and create a data bank for training and testing the developed genetic-artificial neural network.

A total of 855 (eight hundred and fifty-five) images were collected using the mobile application developed. 500 (five hundred) images were selected for the FG-NET database to complement the acquired data in order to train and test the developed age estimation system. Fig. 4 shows sample images from the dataset. Table I shows the age distribution of faces in the database. 


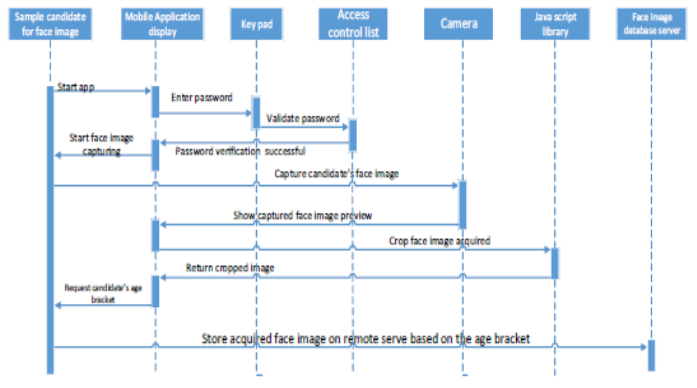

Fig. 2. UML Sequence Diagram for the Face Image Collection Module.

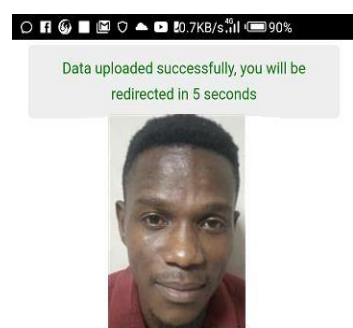

Please select the photo from your phone library and click on save data button.

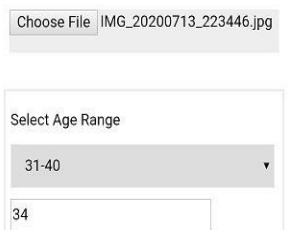

Fig. 3. Mobile App Interface.

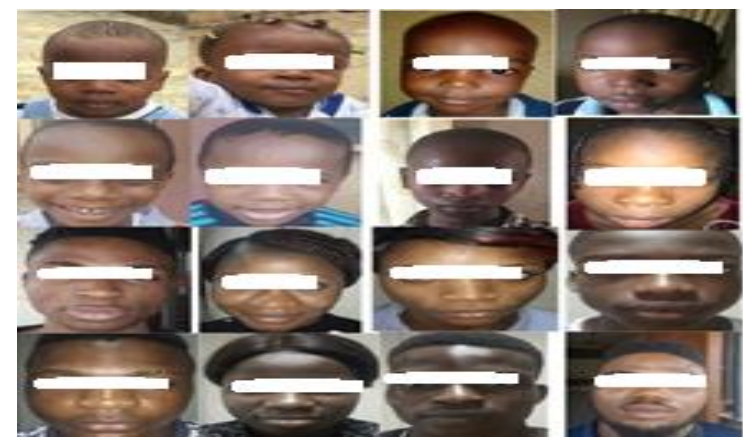

Fig. 4. Sample Images from the Black Face Dataaset.

TABLE I. DATASET Age Distribution

\begin{tabular}{|l|l|l|l|}
\hline Age Groups & Black faces & FG-NET faces & Total \\
\hline 0 to 5 & 109 & 91 & 200 \\
\hline 6 to 10 & 103 & 86 & 189 \\
\hline 11 to 20 & 250 & 113 & 363 \\
\hline 21 to 30 & 118 & 82 & 200 \\
\hline 31 to 40 & 90 & 68 & 158 \\
\hline 41 to 50 & 70 & 39 & 109 \\
\hline 51 to 60 & 57 & 14 & 71 \\
\hline Above 60 & 58 & 7 & 65 \\
\hline Total & 855 & 500 & 1355 \\
\hline
\end{tabular}

\section{B. Simulation Tool}

The age estimation system shown in Fig. 1 was implemented using the MATLAB 2018 object-oriented programming tool. MATLAB was the tool of choice because of its rich computer vision library that makes implementing various techniques and algorithms easy.

\section{Preprocessing}

Images obtained from the mobile app are in-turn stored in age labeled directories on the online image server. During preprocessing the images are converted to grayscale after which the face area are detected using viola jones algorithm and automatically cropped out of the acquired face image. This was to ensure background information that could introduce noise into the system is eliminated. The resulting image is subjected to histogram normalization.

\section{Features Extraction}

The feature extraction technique used in this study is the Local Binary Pattern (LBP). The LBP is used to extract the age information in the face image. The motivation for the choice of the technique is its richness in encoding shape and texture information which are basic theoretical properties that forms the bases of face based age estimation.

Considering a $3 \times 3$ pixels with center pixel (xc,yc) intensity value be $\mathrm{gc}$ and local texture as $\mathrm{T}=\mathrm{t}(\mathrm{g} 0, \ldots, \mathrm{g} 7)$ where gi $(\mathrm{i}=$ $0, \ldots, 7)$ corresponds to the grey values of the 8 surrounding pixels. These surrounding pixels are thresholded with the center value $\mathrm{gc}$ as $\mathrm{t}(\mathrm{s}(\mathrm{g} 0-\mathrm{gc}), \ldots, \mathrm{s}(\mathrm{g} 7-\mathrm{gc}))$ and the function $\mathrm{s}(\mathrm{x})$ is defined in (1). Then the LBP pattern at a given pixel can be obtained using (2) (Lakshmiprabha, 2016).

$$
\begin{aligned}
& S(x)=\left\{\begin{array}{l}
1, x>0 \\
0, x \leq 0
\end{array}\right. \\
& L B P(\mathrm{Xc}, \mathrm{Yc})=\sum_{i=0}^{7} 8(g i-g c) 2^{\mathrm{I}}
\end{aligned}
$$

The LBP feature extracted was further subjected to principal component analysis to reduce the feature set and ensure discriminating features are used as input to the GAANN classifier module. The feature vectors (I) from LBP serves as the training set for the PCA method. M be the total number of images in the training set. The deviation of each image from the mean image is calculated using the (3) and (4).

$\psi=\frac{1}{M} \sum_{n=1}^{M}$ In

$\varphi_{n}=I_{n}-\psi$

The variation among the eigenvectors of the covariance matrix is calculated using (5). The space where all this eigenvectors resides is called as eigenface space or eigenspace. All the training set images are projected into the eigenface space using (5). All training set images are projected to the eigenface space using (6).

$$
\begin{aligned}
& C=\frac{1}{M} \sum_{n=1}^{M} \varphi n \varphi T n=A A^{T} \\
& \omega_{k}=U_{k} \cdot \varphi=U_{k} \cdot(1-\varphi)
\end{aligned}
$$

The Weighted Matrix $\Omega=[\omega 1, \omega 2, \ldots, \omega 0 \mathrm{M}] \mathrm{T}$ is the representation of a training image in the eigenface space. A 
new test image is classified by extracting Gabor and LBP features. It is then mean subtracted using (4) followed by projection onto the eigenface space using (6). Weight matrix of the test image $\Omega \mathrm{T}=[\omega 1, \omega 2, \ldots, \omega 0 \mathrm{M}] \mathrm{T}$ is calculated by projecting test image to eigenspace. This weighted matrix $\Omega T$ is used for classification purpose.

\section{E. Classification using GA-ANN Module}

The GA-ANN is a parallel combination of genetic algorithm and artificial neural network techniques. The GA is aimed at optimizing ANN parameters to generate an optimized ANN object that will in turn be used in predicting the age of a test image. The combination is motivated by the capacity of GA to traverse through a problem space in a timely manner in order to select the fittest candidate solution.

In order to achieve this hybrid system, ANN parameters are encoded as genes in genetic algorithm chromosome. The ANN parameters encoded as genes include the number of hidden layers, the Momentum update (MU), the Momentum update decreasing factor (MU_dec) and the learning rate. Fig. 5 describes a sample gene. The system uses one point crossover technique and Mutation probability (Mp) of 0.1. Other parameters include Number of GA generations (Ngen) and Population size (Ps). The Ngen and Ps used in this study is 100 and 15 respectively in order to ensure the system used for the simulation is not over labored. Candidates' fitness was computed using (7). Fig. 6 shows a sample reproduction mating of two parent in order to form offspring.

Fitness $=\frac{1}{M S E}$

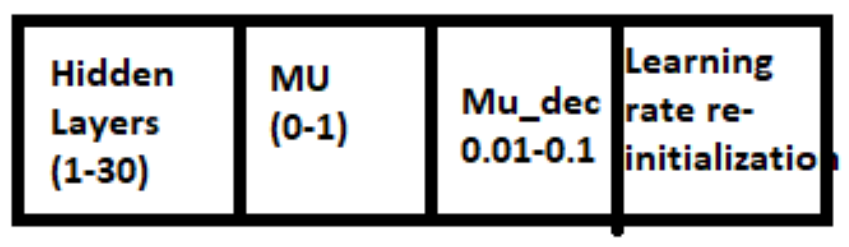

Fig. 5. Sample Chromosome Structure in the Modeled Genes

\begin{tabular}{|c|c|c|c|c|}
\hline \multirow[b]{2}{*}{ Parent A } & \multirow[b]{2}{*}{ Hidden laver $\mathrm{x}$} & & & \\
\hline & & Mu:y & Mu_dec: i & learning rate: $t$ \\
\hline Parent B & Hidden layer x & Mu:y1 & Mu_dec: i1 & Learning rate: $\mathrm{t} 1$ \\
\hline Offspring A & Hidden layer $\mathrm{x}$ & Mu: y1 & Mu_dec: i1 & Learning rate: $\mathrm{t} 1$ \\
\hline Offspring B & Hidden layer x & Mu:y & Mu_dec: i & learning rate: $t$ \\
\hline
\end{tabular}

Fig. 6. Single Point Cross-over Implemented in the Developed Model.
The developed algorithm for the hybridized classifier is shown below.

1. Start

2. Set GA parameters ( $C p, M p$, Ngen and $P s$ )

3. popCount $=$ 0, Fitmax $=$ 0, optimalAnnObject $=N U L L$, genCount $=0$

4. While PopCount $<=$ Ps

5. Generate random values for the ANN parameters encoded into GA chromosome (Number of hidden layer, $\mathrm{Mu}$, and $\left.M u \_d e c\right)$

6. Create parent (ANN object instances) in population

7. Increment the value of PopCount and create new parents by repeating steps 5-6

8. Compute the fitness of each parent (ANN object) using equation 3.

9. While gencount $<=$ Ngen

10. Select parent with high fitness in descending order

11. If fitness (top 1 parent) > fitmax goto 12 else goto 13

12. Fitmax $=$ fitness (top 1 parent) goto 14

13. Fitmax $=$ Fitmax

14. OptimalAnnObject $=$ Parent ( with fitness, fitness)

15. Perform crossover on strongest parents to create new offspring and replace weakest parent with new child created

16. Mutate the population based on mutation probability

17. Compute the fitness of the new population (i.e. new ANN pool)

18. Increament genCount and repeat step (10) to (17) until genCount is equal to number of Ngen

19. optimalAnnObject is assigned GA-ANN with the global minimal solution

20. Return the GA-ANN object for classification

21. Stop

\section{F. Age Estimation System Testing}

Simulation 1: A total of 170 (one hundred and seventy) black faces were used in testing the age estimation system. The age estimation system was trained using the predominantly black-faced database developed for the study. Testing implies that an unknown black face is fed into the optimized GA-ANN module in order to be classified into its appropriate age group. This simulated system is represented as GA-ANN AES-855.

Simulation II: The GA-ANN classifier module was trained with a database with reduced number of black faces. 600 black faces were replaced with faces from FG-NET. This resulted in a training dataset of 1100 FG-NET faces and 255 locally sources black face. 170 black faces were in-turn used to test the trained GA-ANN-based age estimation system. The simulation II is represented as GA-ANN-AES-255. The diagram of testing phase is shown in the Fig. 7 and the testing dataset used is shown in Table II. 


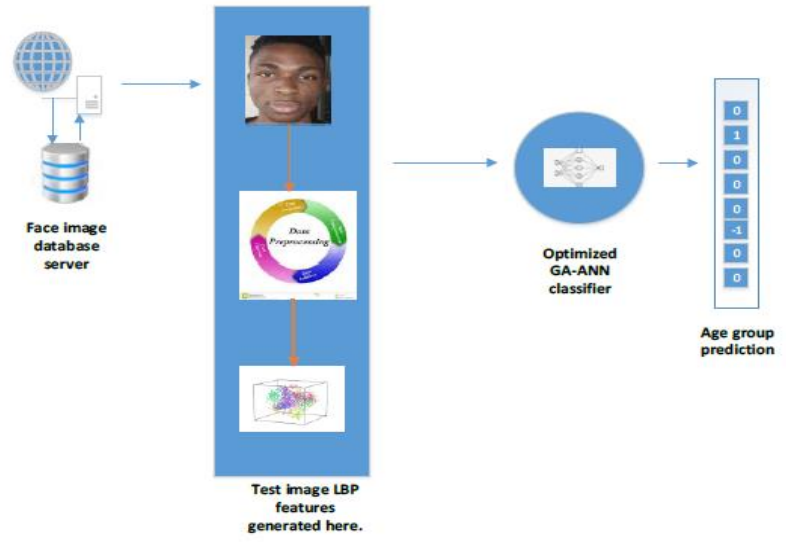

Fig. 7. Diagram of the Testing Phase Architecture.

TABLE II. AgE DistRibUtion OF TEST IMAGES

\begin{tabular}{|l|l|}
\hline Age Groups & Number of test images \\
\hline $0-5$ & 20 \\
\hline $6-10$ & 20 \\
\hline $11-20$ & 30 \\
\hline $21-30$ & 30 \\
\hline $31-40$ & 20 \\
\hline $41-50$ & 20 \\
\hline $51-60$ & 15 \\
\hline Above 61 & 15 \\
\hline TOTAL & 170 \\
\hline
\end{tabular}

\section{G. Performance Evaluation}

The performance of the age estimation system trained using the database containing 855 and 255 black faces, respectively were evaluated using the Correct Classification Rate (CCR). The CCR was computed using (8).

$\mathrm{CCR}=\frac{1}{n} \sum_{i=1}^{n}$ ? $(\mathrm{y}, \mathrm{Y})$

The ' $\delta$ ' is an indicator variable, as such $\delta(y, Y)$ is computed as 1 if $\mathrm{y}=\mathrm{Y}$ and Zero when $\mathrm{y}\langle\mathrm{Y}$. The correct classification rate is used in order to take advantage of its ease of elucidation in discriminant analysis. It's also worth noting that calculating the correct classification rate doesn't need domain-specific data. As a result, this metric may be used to compare the classification accuracy of different models.

\section{RESULT AND DISCUSSION}

This section discusses the results obtained from simulations I and II, respectively, presented in Tabular form. This study categories age into 8 age brackets namely 1-5, 6-10, 11-20, 21$30,31-40,41-50,51-60$ and above 60 . Table III presents the CCR achieved for the age estimation system during simulation I and II, respectively.

The age estimation system simulated with the developed database (GA-ANN-AES-855) outperforms the system simulated using 255 black faces (GA-ANN-AES-255). The aggregate CCR for the GA-ANN-AES-855 is $91.18 \%$ and the CCR for the GA-ANN-AES-255 is $80 \%$.
TABLE III. CORRECT CLASSIFICATION RATE FOR AGE ESTIMATION SYSTEM WITH 855 AND 255 BLACK FACES RESPECTIVELY

\begin{tabular}{|l|l|l|l|l|l|}
\hline & & \multicolumn{2}{|l|}{ GA-ANN-AES-855 } & \multicolumn{2}{|l|}{ GA-ANN-AES-255 } \\
\hline AGE & $\begin{array}{l}\text { Test } \\
\text { Image } \\
\text { s }\end{array}$ & $\begin{array}{l}\text { Correct } \\
\text { Classificati } \\
\text { on }\end{array}$ & $\begin{array}{l}\text { CCR } \\
(\%)\end{array}$ & $\begin{array}{l}\text { Correct } \\
\text { Classificat } \\
\text { ion }\end{array}$ & $\begin{array}{l}\text { CCR } \\
(\%)\end{array}$ \\
\hline $0-5$ & 20 & 18 & 90.00 & 14 & 70.00 \\
\hline $6-10$ & 20 & 18 & 90.00 & 16 & 80.00 \\
\hline $11-20$ & 30 & 30 & 100.00 & 27 & 90.00 \\
\hline $21-30$ & 30 & 26 & 86.67 & 24 & 80.00 \\
\hline $31-40$ & 20 & 19 & 95.00 & 17 & 85.00 \\
\hline $41-50$ & 20 & 18 & 90.00 & 16 & 80.00 \\
\hline $51-60$ & 15 & 13 & 86.67 & 11 & 73.33 \\
\hline Above 61 & 15 & 13 & 86.67 & 11 & 73.33 \\
\hline $\begin{array}{l}\text { TOTAL/AG } \\
\text { G }\end{array}$ & $\mathbf{1 7 0}$ & $\mathbf{1 5 5}$ & $\mathbf{9 1 . 1 8}$ & $\mathbf{1 3 6}$ & $\mathbf{8 0 . 0 0}$ \\
\hline
\end{tabular}

This result is in agreement with [8]. The reason for this is the other race effect. The more the population of a race in the training dataset, the better the recognition performance with a testing set of the same race. The result could also be seen to be in agreement with the discoveries in [5] that indicated that insight from racial information could affect the awareness of memory processes. In this case, age estimation, a computerbased recognition process, is being affected by the racial information in the training data.

Also, the trend in the results showed that the highest correct classification rates were achieved in the age brackets of 11-20. The age bracket has the highest number of images in the dataset - 363 images. This indicates that the discriminating ability of the encoded facial features in discerning age is improved with the quantity of images in the training dataset. This aligns with the discoveries of Oladele [20]. The AES performs less than expected when tested with faces that are not popular in the training dataset.

Fig. 8 shows the chart of the CCR of GA-ANN-AES-855 and GA-ANN-AES-255, respectively.

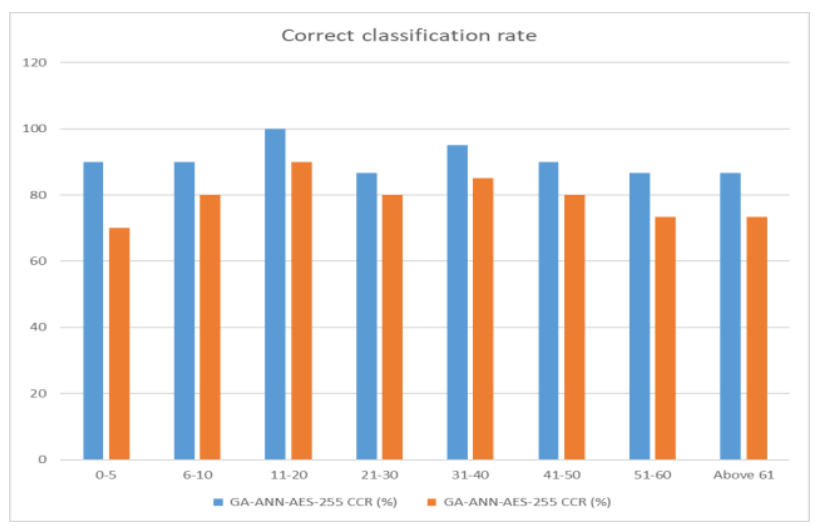

Fig. 8. Correct Classification rate: GA-ANN-AES-855 and GA-ANN-AES255. 
The result of the experimentation was further subjected to inferential statistics using one-way-ANOVA. The result is as shown.

The result, after subjecting Table II which showed the CCR of GA-ANN-AES-255 and GA-ANN-AES-855 to statistical analysis:

$\mathrm{H}_{0}$ : No significant improvement in the CCR of GA-ANNAES-855 over GA-ANN-AES-255

$\mathrm{H}_{1}$ : There is significant improvement in the CCR of GAANN-AES-855 over GA-ANN-AES-255

Significance level $=0.05$

Equal variances were assumed for the analysis.

Factor Information

Factor Levels Values

Factor 2 GA-ANN-AES-255, GA-ANN-AES-855

Analysis of Variance

$\begin{array}{llll}\text { Source DF } & \text { Adj SS } & \text { Adj MS F-Value P-Value } \\ \text { Factor 1 544.6 } & 544.64 & 16.56 & 0.001 \\ \text { Error } 14 & 460.4 & 32.89 & \\ \text { Total } 15 & 1005.1 & & \end{array}$

The P-value (0.001) showed in the result of the analysis is less than 0.05 . Hence, the alternative hypothesis is accepted and the null hypothesis is rejected. This implies that the improvement in correct classification rate of GA-ANN-AES855 over GA-ANN-AES-255 is statistically significant.

\section{CONCLUSION AND RECOMMENDATION}

The study examines the effect of other-race effect on automatic age estimation. In order to achieve this, we developed a predominantly black face database that contains 855 black faces complemented with 500 FG-NET faces. The developed database was used in training a GA-ANN age estimation system. A second scenario is simulated, with the GA-ANN-based age estimation system trained with 255 black face images and 1100 FG-NET faces. Both systems were tested with 170 black faces to query if the heightened number of black faces in the developed image database ensures a better correct classification rate.

The study showed that the GA-ANN-AES-855 outperformed the GA-ANN-AES-255 with a CCR of $91.18 \%$ compared to the $80.00 \%$ shown by the GA-ANN-AES-255. Inferential statistics to show the improvement is statistically significant using one way ANOVA was conducted. The result of the statistical evaluation established that the improvement was significant and hence showed that the other-race effect could affect the performance of an age estimation system.

This study recommends the following:

1) The aggregation of various black face databases to form a huge face image database that can foster the use better techniques that perform when image data volume is much.
2) Deployment of the developed genetic algorithmartificial neural network age estimation model on mobile phone platform to ensure age verification can be carried out using application built using such model.

3) The development of age estimation algorithms that will be robust enough to minimize other age effect in age estimation.

\section{REFERENCES}

[1] R. Angulu, J. R. Tapamo, and A. O. Adewumi, "Age-group estimation using feature and decision level fusion," The Computer Journal, vol. 62, no. 3, pp. 346-358, 2018.

[2] O. Oladipo, I. P. Osamor, V. C. Osamor, T. N. Abiodun, A. O. Omoremi, M. O. Odim, and R. H. Ekpo, "Face-age modeling: A pattern recognition analysis for age estimation," 2019 IEEE International Conference on Bioinformatics and Biomedicine (BIBM), 2019.

[3] D. Akinyemi and O. Onifade, The impact of indigenous ethnicity on facial image analysis, Academia Letters. Vol 2, pp.2-7. 2021.

[4] J.L.Yaros, D.A. Salama, D. Delisle, M.S. Larson, B.A. Miranda and M.A.Yassa, "A memory computational basis for the other-race effect. Scientific reports, Vol 9, pp.1-11, December 2019.

[5] J. Chen and X. Zhu, "The cross-race effect on face recognition and judgments of learning," Proceedings of the 3rd International Conference on Culture, Education and Economic Development of Modern Society (ICCESE 2019),vol. 310, pp. 672-675, 2019.

[6] M. Stelter and J. Degner, "Investigating the other-race effect in working memory," British Journal of Psychology, vol. 109, no. 4, pp. 777-798, 2018.

[7] J. R. Collova, N. Kloth, K. Crookes, N. Burton, C. Y. Chan, J. H. Hsiao, and G. Rhodes, "A new other-race effect for gaze perception.," Journal of Experimental Psychology: Human Perception and Performance, vol. 43, no. 11, pp. 1857-1863, 2017.

[8] P. J. Phillips, F. Jiang, A. Narvekar, J. Ayyad, and A. J. O'Toole, “An other-race effect for face recognition algorithms," ACM Transactions on Applied Perception, vol. 8, pp. 1-11, January 2011.

[9] J.D. Akinyemi and O.F.W Onifade. "A computational face alignment method for improved facial age estimation." In 2019 15th International Conference on Electronics, Computer and Computation (ICECCO), pp. 1-6. IEEE, 2019.

[10] G. Guo and G. Mu, "A framework for joint estimation of age, gender and ethnicity on a large database," Image and Vision Computing, vol. 32, pp. 761-770, 2014.

[11] K.-Y. Chang, C.-S. Chen, and Y.-P. Hung, "Ordinal hyperplanes ranker with cost sensitivities for age estimation," Conference on Computer Vision and Pattern Recognition, August 2011.

[12] K.k. Kamarajugadda and T.R Polipalli, Extract features from periocular region to identify the age using machine learning algorithms. Journal of medical systems. Vol. 3, pp.1-5, July 2019.

[13] H. Han, C. Otto, and A. Jain, "Age estimation from face images: Human vs. machine performance," in Biometrics (ICB), 2013 International Conference, pp. 1-8, June 2013.

[14] R. Angulu, J.R. Tapamo, and A.O. Adewumi, Age estimation via face images: a survey. EURASIP Journal on Image and Video Processing, pp.1-35, June 2018.

[15] K. Chen, K. Jia, H. Huttunen, J. Matas and J.K Kämäräinen, "Cumulative attribute space regression for head pose estimation and color constancy," Pattern Recognition, Vol. 87, pp.29-37. 2019.

[16] A. Demontis, B. Biggio, G. Fumera, and F. Roli, "Super-sparse regression for fast age estimation from faces at test time," in Image Analysis and Processing ICIAP 2015, pp. 551-562, Springer, 2015.

[17] X. Wang and C. Kambhamettu, "Age estimation via unsupervised neural networks," in Automatic Face and Gesture Recognition (FG), 2015 11th IEEE International Conference and Workshops on, vol. 1, pp. 1-6, May 2015.

[18] S. Hosseini, S.H. Lee, H.J. Kwon, H.I. Koo and N.I. Cho, "Age and gender classification using wide convolutional neural network and Gabor filter," In 2018 International Workshop on Advanced Image Technology (IWAIT), pp. 1-3., January 2018.

[19] D. Yi, Z. Lei, and S. Z. Li, "Age estimation by multi-scale convolutional network," in Computer Vision-ACCV, pp. 144-158, 2015. 
[20] M. Oladele, E. Omidiora, and A. Afolabi, "A face-based age estimation system using back propagation neural network technique," British Journal of Mathematics \& Computer Science, vol. 13, pp. 1-9, 2016.

[21] E. Omidiora, M. Oladele, T. Adepoju, A. Sobowale, and O. Olatoke, "Comparative analysis of back Propagation neural network and selfOrganizing feature map in Estimating age groups using facial features," British Journal of Applied Science \& Technology, vol. 15, pp. 1-7, 2016.

[22] S. Chen, C. Zhang, M. Dong, J. Le, and M. Rao, "Using ranking-cnn for age estimation," 2017 IEEE Conference on Computer Vision and Pattern Recognition (CVPR), 2017.

[23] K. Li, J. Xing, W. Hu, and S. J. Maybank, "D2c: Deep cumulatively and comparatively learning for human age estimation," Pattern Recognition, vol. 66, pp. 95-105, 2017.

[24] Z. Qawaqneh, A. A. Mallouh, and B. D. Barkana, "Age and gender classification from speech and face images by jointly fine-tuned deep neural networks," Expert Systems with Applications, vol. 85, pp. 76-86, 2017.

[25] Z. Ji, C. Lang, K. Li, and J. Xing, "Deep age estimation model stabilization from images to videos," 2018 24th International Conference on Pattern Recognition (ICPR), 2018.
[26] S. Zaghbani, N. Boujneh, and M. S. Bouhlel, "Age estimation using deep learning," Computers \&amp; Electrical Engineering, vol. 68, pp. 337-347, 2018.

[27] M. Duan, K. Li, C. Yang, and K. Li, "A hybrid deep LEARNING Cnnelm for age and gender classification," Neurocomputing, vol. 275, pp. 448-461, 2018.

[28] J. Wan, Z. Tan, Z. Lei, G. Guo, and S. Z. Li, "Auxiliary demographic information assisted age estimation with cascaded structure," IEEE Transactions on Cybernetics, vol. 48, no. 9, pp. 2531-2541, 2018.

[29] R. Angulu, J. R. Tapamo, and A. O. Adewumi, "Age estimation with local ternary directional patterns," Image and Video Technology, pp. 421-434, 2018.

[30] S. H. Nam, Y. H. Kim, N. Q. Truong, J. Choi, and K. R. Park, "Age estimation by SUPER-RESOLUTION reconstruction based on adversarial networks," IEEE Access, vol. 8, pp. 17103-17120, 2020.

[31] N.F Hasan and S. Q. Mahdi. "Facial Features Extraction Using LBP for Human Age Estimation Based on SVM Classifier." In 2020 International Conference on Computer Science and Software Engineering (CSASE), pp. 50-55. IEEE, 2020. 